Proceedings of the 2011 Winter Simulation Conference

S. Jain, R. R. Creasey, J. Himmelspach, K. P. White, and M. Fu, eds.

\title{
DYNAMIC MORTALITY SIMULATION MODEL INCORPORATING RISK INDICATORS FOR CARDIOVASCULAR DISEASES
}

\author{
Jocimara S. Ferranti \\ Paulo J. de Freitas Filho \\ Postgraduate Program in Computer Science \\ Dept. of Informatics and Statistics \\ Federal University of Santa Catarina \\ Caixa Postal 476 - 88.040-900 \\ Florianópolis/SC - Brazil
}

\begin{abstract}
This article describes a dynamic-fuzzy simulation model and proposes an extension to it. The model represents a person's physiological capacity throughout life and simulates the occurrence of risk events from birth until death, including a representation of the process of recovering health after it has been impacted by a risk event. The expanded model incorporates cardiovascular risk factors in order to reproduce curves plotted from real mortality data from a specific population whose cause of death was cardiovascular diseases. By adjusting the parameters, it proved possible to reproduce mortality curves from populations with specific characteristics such as hypertension, obesity and physical activity levels. A simulation model that is capable of focusing on specific populations makes it possible to test alternative intervention designed to reduce the mortality caused by specific diseases, thereby contributing to improved quality-of-life for populations and to cost savings for both public and private health care systems.
\end{abstract}

\section{INTRODUCTION}

For a very long time, humans have been studying the aging process and those elements of it that make people more and more vulnerable to health problems as time passes. While the advances made by modern medicine have led to an increase in populations' life expectancies, the quest for longevity and quality of life still consumes very significant resources (Hayflick 1997).

Certain types of disease can be identified as causing the largest numbers of deaths in modern times. Diseases related to the cardiovascular system and cancers are responsible for a great deal of expenditure by both the public and private sectors. These costs are by no means restricted to the health care sector. While the incidence of these types of disease continues to be related to a range of indicators that express a person's lifestyle habits and health conditions, changing lifestyle habits are equally capable of worsening or improving the incidence among populations.

The aim of this study is to expand the aging and death simulation model proposed by Schwaab and de Freitas Filho (2009), by including risk factors for cardiac diseases, in order to improve understanding of the interaction between these factors and the occurrence of age-related diseases. The hope is that the output of such a model could reproduce real-life data from populations with specific pathological characteristics.

In addition to facilitating the study of populations of people with similar characteristics in terms of risk situations, this research may also improve our understanding of the effects that changes to these parameters

has on given populations, thereby contributing to increasing people's quality of life and reducing the cost of both public and private health care. This type of study provides a basis for planning preventative strategies designed to avoid future problems. 


\section{Ferranti and Freitas Filho}

\section{METHODOLOGY}

The methods employed to achieve the goal of expanding the earlier model were primarily based on the System Dynamics methodology (Forrester 1961). System Dynamics is a modeling technique that provides a common foundation that can be applied to any type of system when it is necessary to understand and to influence the way in which things change over time (Forrester 1998). Classified as a modeling technique for learning, it aids in achieving deeper understanding of the behavior of systems, with the objective of identifying problematic points at which a range of alternative options can then be tested (Ford 1999).

The first academic papers dealing with System Dynamics only appeared after the technique had been applied to corporate, administrative and even urban problems for a long time (Radzicki and Taylor 1997, Forrester 1961, Forrester 1969). The technique is not, however, limited to these types of systems, but can also be used in areas such as medicine, the environment, economics and politics, since it combines the theory, methods and philosophy needed to analyze the behavior of systems (Forrester 1998).

According to this methodology, dynamic simulation models are constructed from 3 components. Stocks, which are also known as levels, convey the idea of storage, representing the accumulation of entities and the state in which a given variable is over time. Flows represent the processes through which entities pass, both when being added to stocks and when being removed from them, and which determine changes to stock levels. Finally, converters are auxiliary elements that modulate the flows as they transform the stocks and represent rates of change, constants and calculations in general. In combination, these three elements form the basic structure of dynamic simulation models, which are also known as flow diagrams (Deaton and Winebrake 2000).

The model proposed here has been implemented using the Stella platform (isee Systems 2011), which is a System Dynamics-oriented tool for continuous dynamic simulation that provides the elements necessary to construct flow diagrams and causal loop diagrams, thereby improving understanding of the problem being studied.

As with any model, the model proposed here has limits and it is important to define them clearly. This model does not provide evidence in support of theories about aging and its peculiarities, although it could be classified within one of these theories. Furthermore, this is not a tool to substitute existing methods of population study, but an additional option.

\section{STOCHASTIC MODEL OF AGING AND DEATH}

The model on which the current work is based is a stochastic simulation tool designed to aid with the study of the processes of human aging through continuous dynamic modeling and fuzzy modeling (Schwaab and de Freitas Filho 2009). The simulation is primarily based on a model of aging and death proposed by Hargrove (1998), and the core elements of the model proposed by Schwaab and de Freitas Filho (2009), that is shown in Figure 1 draw on quantitative theories of aging that make it possible to simulate risk events that may take place during a person's lifetime, tracing both effects that are favorable and effects that are detrimental to the subject's survival.

In the model shown in Figure 1, total and relative capacities represent a person's physical functions. Total Capacity is made up of three components: (1) Function, which is related to personal autonomy, (2) Reserves, which can be considered as the quantity of energy that a person has while alive (3) and Aging, which, in contrast with Function and Reserves, reduces a person's total capacity. The state of each of these elements is defined by the growth rate, reserve rate and rate of aging, respectively. These rates are a function of the interaction between a person's genetics and the environment in which they live. Relative Capacity, in turn, is derived from Total Capacity.

In addition to these elements, the model also expresses the occurrence of problems during a person's lifetime. These may be accidental or could be a consequence of age. The probability of these problems occurring is determined by Age-Dependent Factors, Age-Independent Risk and Relative Capacity, which are calculated using the Monte Carlo method (Hammersley and Handscomb 1964). When a problem does 


\section{Ferranti and Freitas Filho}

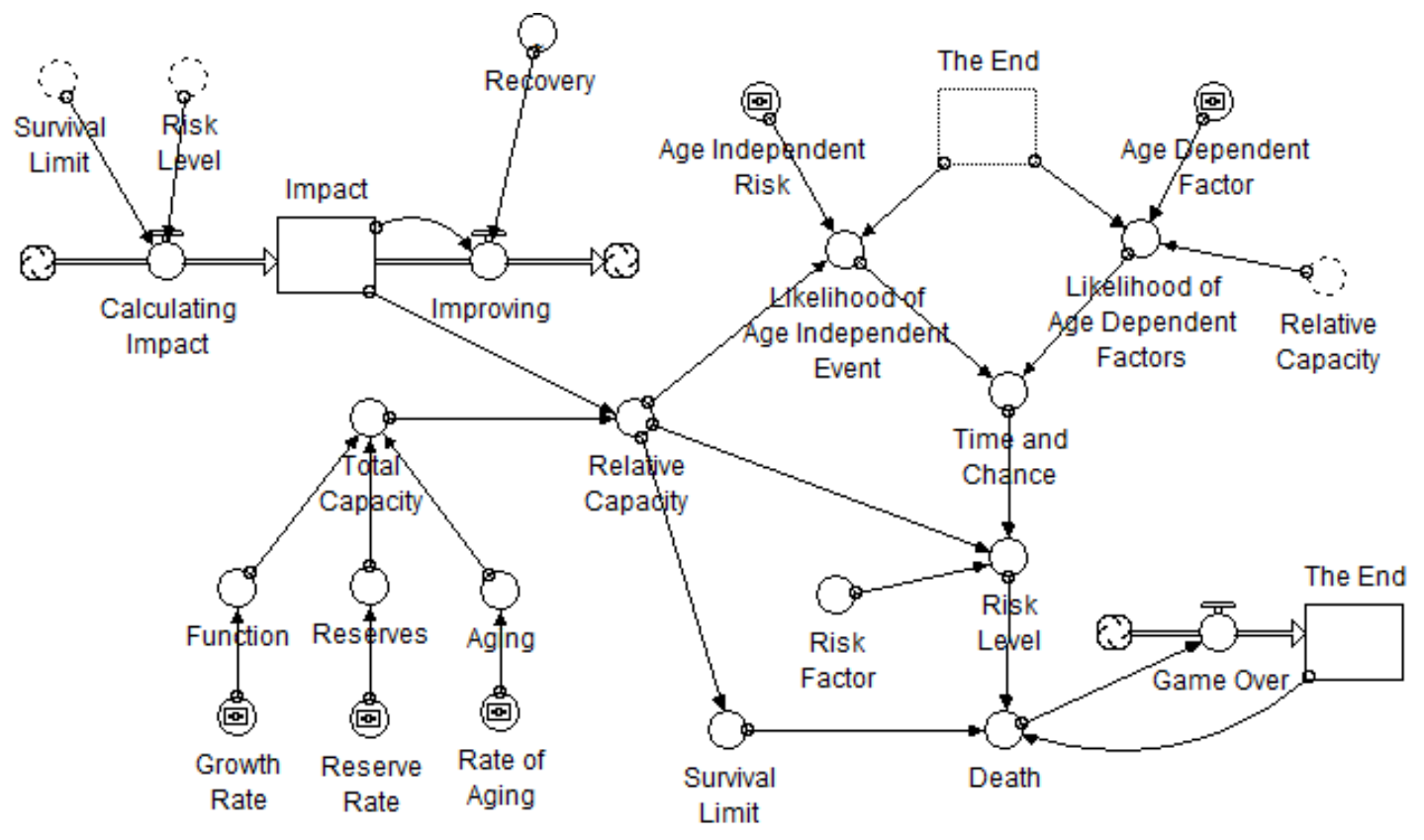

Figure 1: Stochastic model of aging and death (Schwaab and Freitas Filho 2009).

occur, the Risk Level determines the event's intensity and this is then compared with the person's Survival Limit to determine whether or not they will survive the episode.

If they do survive, the recovery module determines the extent to which their health is affected and the manner in which recovery will occur over time. The module does not only calculate recovery on the basis of the event's impact, but also on the basis of the person's age, since the more advanced a person's age, the lower their capacity to recover (Hayflick 1997).

\subsection{Parameters of the Model}

\subsubsection{Growth Rate}

Growth Rate $(G R)$ determines the behavior of a person's growth function, representing functional capacity from birth to death. It increases to a maximum value and then remains constant for the rest of the person's life. The growth function is labeled Function $(F)$ and is defined by the following equation (1), where $t$ represents the simulation period - i.e. the age of the person whose life is being simulated.

$$
F(t)=40+(40 *(1-\exp (-G R * t)))
$$

As will be observed from the equation, the minimum possible value of function $F$ is 40 and the result of the exponent approaches zero as time passes, which in turn causes $F$ to approach 80 . It can therefore be concluded that the greater the value of $G R$, the faster the function will reach its maximum level.

\subsubsection{Reserve Rate}

The Reserve Rate $(R R)$ determines the behavior of a function that represents the subject's vital energy. Defined by the expression below (2), the Reserves $(R)$ function is characterized by growth up until approximately 20 years of age, followed by a gradual decline from that point on until death.

$$
R(t)=10+(10 *(1-\exp (-R R * t))-10 *(1-\exp (-0,04 * t))
$$




\section{Ferranti and Freitas Filho}

In this equation, the greater the Reserve Rate, the higher the point at which the function $R$ peaks and the earlier this maximum point is reached. However, it is important to point out that, the greater the value of $R R$, the earlier the phase during which the value of $R$ declines will begin, meaning that the energy of a person with high $R R$ will begin to reduce earlier than expected.

\subsubsection{Rate of Aging}

The Rate of Aging $(R A)$ determines the behavior of a function (3) with the same name. The function represents a process that begins when people reach their maximum level of development and which, according to Hargrove (1998), starts after 25 years of age.

$$
A(t)= \begin{cases}90 *(1-\exp (-R A *(t-25))), & \text { if } t \geq 25 \\ 0, & \text { if } t<25\end{cases}
$$

In this expression, it will be observed that the rate at which the result of this function increases is more accentuated for larger values of $R A$.

Taken together, Function, Reserves and Aging give a person's Total Capacity, as defined by the equation below (4). Whereas $F$ and $R$ increase the magnitude of the result of the function, $A$, together with other adjustment factors that are dependent on age $(t)$, causes a reduction in $T C$, particularly after 25 years of age, when the value of function $A$ is no longer zero.

$$
T C(t)=F(t)+R(t)-A(t)-(0,1 * t)-10 *(1-\exp (-0,01 * t))
$$

Finally, in order to calculate a person's effective fitness in the event of a risk, Relative Capacity $(R C)$ is derived from $T C$ using the equation below (5), where $I$ is the Impact that a risk event would have on the person's health if it were to occur. The value of $I$ is the output of an Impact and Recovery Module that will be described in item 3.2 below.

$$
R C(t)=\frac{T C(t) *(1-I(t))}{87,67} * 100
$$

\subsubsection{Age-Dependent Factors}

Age-Dependent Factors $(A D F)$ represent a person's vulnerability to unpredictable risks because of agerelated factors. The result defines the probability of this type of risk event occurring at some point during a person's life.

$$
A D E(t)=\text { MonteCarlo }\left(\frac{A D F * t}{R C(t)}\right)
$$

According to this equation (6), a MonteCarlo function determines whether or not an Age-Dependent Event $(A D E)$ occurs. This function generates a series of zeroes and ones depending on a given probability, which itself is set as one of the function's parameters. As would be expected, the probability of an Age-Dependent Event occurring increases as time passes.

\subsubsection{Age-Independent Risk}

Age-Independent Risk $(A I R)$ also represents people's vulnerability to unpredictable risks, but in the case of this parameter, the risks are external and totally independent of age.

$$
A I E(t)=\text { MonteCarlo }\left(\frac{2 * A I R}{R C(t)}\right)
$$




\section{Ferranti and Freitas Filho}

A MonteCarlo function also defines whether or not an Age-Independent Event (AIE) occurs, as shown in the equation above (7) and while it is not dependent on a person's age, the probability that an event of this type will occur also increases as time passes, because Relative Capacity reduces. Together, these two factors define whether a person will or will not suffer a Risk Event Occurrence (REO), irrespective of whether that event is dependent on or independent of age.

$$
R E O(t)=A D E(t) \vee A I E(t)
$$

\subsubsection{Risk Level}

In the event of a Risk Event Occurrence, the Risk Level $(R L)$ defines the effect that the event will have on a person's health, defined according to the following equation (9).

$$
R L(t)=\operatorname{Random}(0, R F(t) * R C(t))
$$

In this equation, the Risk Factor $(R F)$ combines variables related to the health conditions of the population and is involved in determining the extent to which an event will affect a person's health. The effect of $R F$ is different at different ages, becoming more intense as age increases. The value of $R F$ is in the range of 0.525 to 0.7 , which represents a health status that is considered reasonable (Hall and Zwemer 1979).

The Survival Limit $(S L)$ defines the maximum effect that a person can suffer and is defined by the following equation (10).

$$
S L(t)=0.45 * R C(t)
$$

If the Risk Level of the event suffered is greater than the person's Survival Limit then they die. However, if the effect is below this limit, the person's Total Capacity is reduced, representing the impact that the event has on their health.

\subsection{Impact and Recovery Module}

The Impact and Recovery Module represents a person's capacity to recover from an impact that puts their health and survival at risk. It is designed to determine the speed with which they are able to recover, based on their age and the intensity of the impact they have suffered.

The effects of a risk event have an Impact on the subject's capacity for survival, in order to simulate the sequelae left by the event. These possibly reduce over time, as Recovery progresses. Furthermore, Recovery is also dependent on the age of the person being modeled, since the more advanced their age, the lower their capacity for Recovery. The Impact and Recovery Module therefore comprises a fuzzy inference machine (Zadeh 1965) whose inputs are the variables Age and Impact and whose output is Recovery of the subject's health.

Before calculating Recovery, it is first necessary to define the Impact suffered (I), i.e., the extent to which the risk event has affected the person's health.

$$
I(t)=I(t-1)+(C I(t)-I M P(t))
$$

In the expression above (11), $t$ is current simulation time and $t-1$ is the immediately preceding time. The value of $C I$ defines the extent to which the event impacts on the person's health, increasing the value of $I$. In contrast, IMP represents the person's Improvement after a Risk Event Occurrence and as such reduces the value of $I$. Their magnitudes are defined by the following two equations (12) and (13). In the second expression (13), a person's Recovery is given by the output of the fuzzy inference machine and is represented by $R E C$. 


$$
\begin{gathered}
C I(t)=\frac{R L(t)}{S L(t)} \\
I M P(t)=R E C(t) * I(t)
\end{gathered}
$$

The input variables Age and Impact are used to define the value of $R E C$. Age is expressed as a number in the range of zero to 100 years and then split into four fuzzy sets - children, young people, adults and the elderly - determined using a trapezoidal membership function. The variable Impact is defined along a scale from zero to 1 and also has four fuzzy sets - weak, mild, moderate and strong - distributed in an approximately uniform manner along the variable's support. Since this input has a greater degree of imprecision, its fuzzy sets were defined using sigmoidal membership functions for the terms "weak" and "strong" and using the product of two sigmoidal functions for the terms "mild" and "moderate" (Klir and Yuan 1995). Finally, the variable Recovery was defined in a similar manner, using sigmoidal functions and the product of two sigmoidal to classify the values along a support from zero to 1, comprising the fuzzy sets stable, slow, moderate and fast.

The rule base takes account of the fact that a person's vital functions reduce as they get older, meaning that their capacity to recover also reduces with age (Table 1). This means that the process of improvement becomes ever slower.

Table 1: Rule base for Recovery. Statements of the type IF $a$ AND $b$ THEN $c$ were used to create rules with the following format: IF Age is child AND Impact is weak THEN Recovery is fast.

\begin{tabular}{r|llll}
\hline & \multicolumn{4}{|c}{ Impact } \\
Age & Weak & Mild & Moderate & Strong \\
\hline Child & Fast & Fast & Moderate & Slow \\
Youth & Fast & Fast & Moderate & Moderate \\
Adult & Moderate & Moderate & Slow & Slow \\
Elderly & Slow & Stable & Stable & Stable \\
\hline
\end{tabular}

Figure 2 shows the output surface obtained using the following fuzzy system parameters: product for t-norm and implication, maximum for aggregation and smallest of maximum for defuzzyfication. It will be observed that the greater the values of Age and Impact, the lower the value of Recovery.

\section{EXPANSION OF THE SCHWAAB AND FREITAS FILHO MODEL (2009)}

The model proposed in this paper is an expansion of a model created by Schwaab and de Freitas Filho (2009) and is designed to determine a person's probability of death according to their age, health status and lifestyle habits.

Cardiovascular diseases rank as one of the principal causes of death globally and that is why they were chosen as the focus of this proposed model, which is designed to simulate the behavior of a population that has a series of factors habitually used to screen for this potential cause of death. The extension to the model, which is illustrated in Figure 3, incorporates elements taken from the Geller tables, which make it possible to use variables such as sex, age and risk indicators to determine the probability that some type of health problem will occur during a person's life (Hall and Zwemer 1979).

A Compound Risk Factor $(C R F)$ is an expression of a person's level of risk of a given cause of death; in this case a cardiovascular disease. Its value represents the probability that a person with certain characteristics will die of the cause of death in question. 


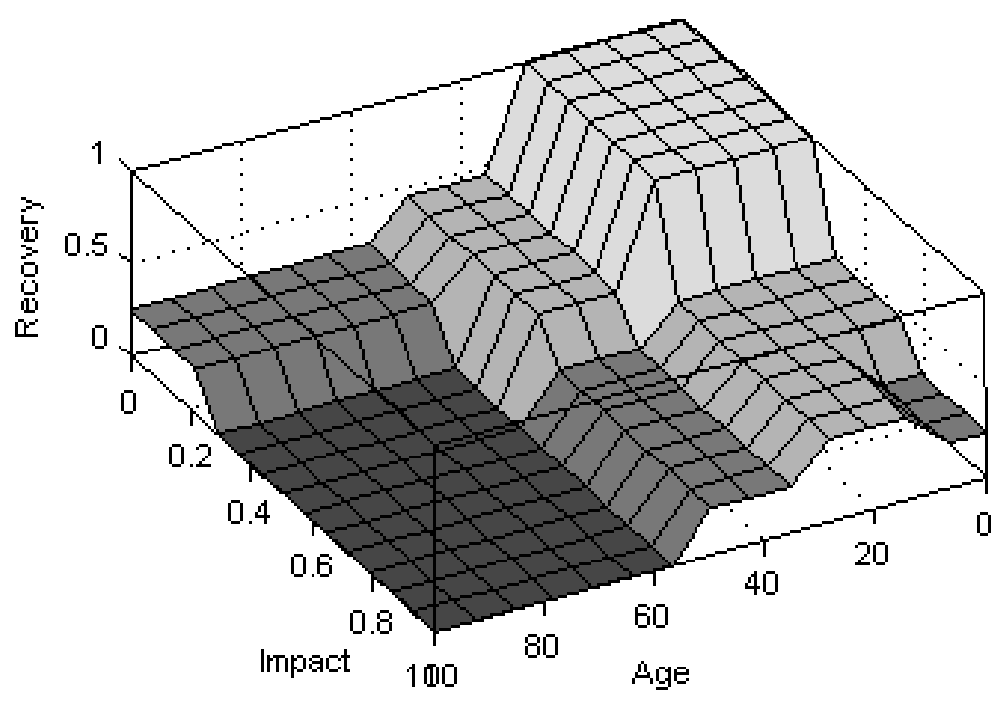

Figure 2: Output surface - Recovery.

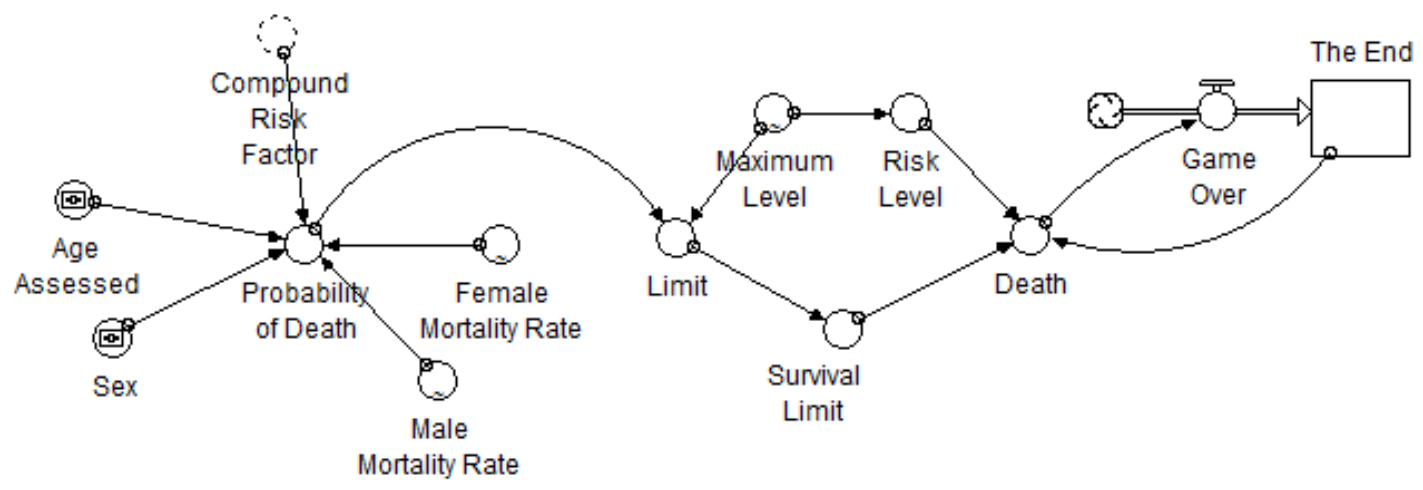

Figure 3: Proposed expansion of model.

\subsection{Implementation of the Compound Risk Factor Module}

The $C R F$ is calculated on the basis of individual risk indicators that are applicable to the cause of death in question and which represent a population's health characteristics and lifestyle habits. The inputs for this calculation are the values of each indicator, by age and sex of the person whose risk is being modeled. The indicators included for cardiovascular diseases are: systolic pressure, diastolic pressure, cholesterol, diabetes, physical activity, smoking and obesity.

The value of each risk indicator, also known as the factor's relative risk, is calculated from the odds ratio between the probability of death due to the disease in question, for members of a subset with a given risk indicator, and the probability of death from the same cause for all members of the population.

The risk indicators are combined in the following manner in order to calculate a person's $C R F$ : all indicators with a risk factor ratio of $<1$ are multiplied together and the product is added to the sum of all indicators with a factor of $>1$. The number of risk indicators with a factor of $>1$ is then subtracted from the result of the last addition (Hargrove 1998).

As will be observed in Figure 3, the $C R F$ is used to calculate a person's Probability of Death $(P D)$. Depending on the sex that is input, the model will multiply the $C R F$ by the corresponding probable mortality 


\section{Ferranti and Freitas Filho}

rate, resulting in a new probability of death that is increased or decreased according to the subject's lifestyle habits, which are reflected in their $C R F$.

The subject's probability of death is then used to calculate their Survival Limit for the age group in which they are during the simulation. Originally, the Survival Limit was calculated simply on the basis of the subject's Relative Capacity, as shown in the $S L$ equation (10) above. The alteration proposed here is to vary the Survival Limit in accordance with the calculated probability of death, which substitutes the constant originally used to multiply the Survival Limit with a coefficient derived from the subject's probability of death.

The objective of this modification is to make the Survival Limit function reflect the impact that $C R F$ has on survival, i.e., the greater the probability of death, the lower the Survival Limit, and vice versa.

The starting point for calculating the new Survival Limit coefficient is the value of $R F$, which is treated as certain death for a person in the age group in question. The Limit $(L)$ defines the multiplier that will then set the subject's Survival Limit, according to the expression below (14), where $R F$ and $P D$ are the Risk Factor and the subject's Probability of Death, respectively.

$$
L(t)=\frac{(R F(t) * 100)-(R F(t) * P D(t))}{100}
$$

Finally, the formula for Survival Limit given earlier was altered as shown in the following equation (15).

$$
S L(t)=L(t) * R C(t)
$$

The objective of this calculation was to achieve a distance between the curves for Risk Factor and Survival Limit, when superimposed, that corresponds to the probability of death calculated previously.

\section{RESULTS}

Data from two different sources were used to validate the results of the expanded model: (1) the EPIDOSO project, which is a prospective epidemiological study of elderly people conducted by the Center for Aging Research at the Federal University of São Paulo (Ramos 1998) and which provides data on the characteristics of the population; and (2) the SEADE Foundation which is the State of São Paulo's data processing agency and which provided mortality data on this population, with dates and causes of death (SEADE 2011). The two datasets were sorted into case studies, according to the population's characteristics and mortality rates were classified by age groups.

Each complete simulation using the model represents one person and the results of the simulation represent that person's lifetime. It was necessary to adjust the fit of the parameters in order to achieve acceptable results for all case studies in which the cause of death was cardiovascular diseases. These values were defined taking into account the sensitivity reported by an experimental study that monitored all of the parameters needed for the original model and observation of the resulting data.

The objective was not to achieve results that were identical to the real data, but to achieve an approximation that was within acceptable margins of error. The real population data used to validate the model was broken down according to certain characteristics in order to define specific case studies that were then analyzed in order to aid understanding of the effect that the risk indicators have on the life expectancy and mortality rate of the population. Results were verified by calculating confidence intervals for each age group. The results are illustrated in Figures 4 to 7 below, each of which show the curve generated by the expanded simulation model, the curve traced by the real-life data and the confidence interval limits, adopting $\alpha=0.05 \%$.

Figure 4 presents results of the proposed model for the validation case with the following characteristics: male, free from high blood pressure, free from diabetes, not obese and does not practice physical activity. Data on smoking and cholesterol not given. 


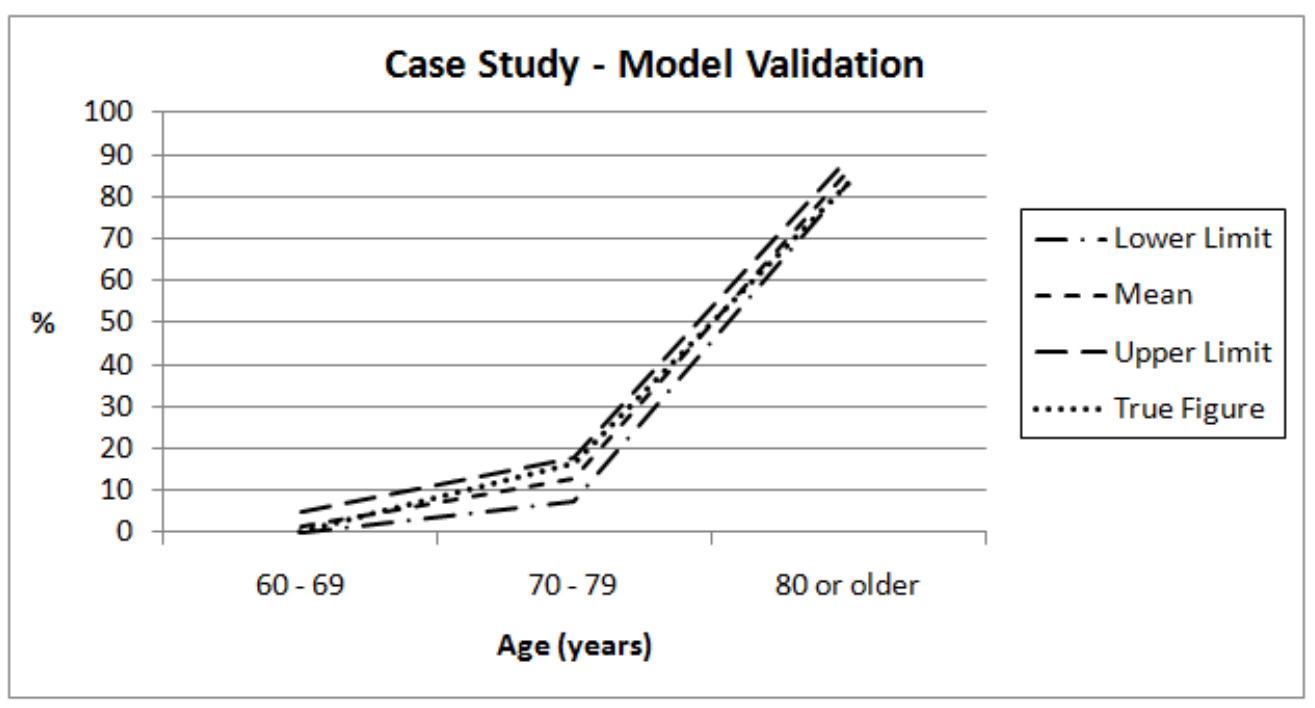

Figure 4: Results of the proposed model for a case with the following parameters: $G R=0.14, A D F=4$, $R R=0.05, A I R=100$ and $R A=0.018$.

The model was judged to be valid on the basis of the graph shown in Figure 4, since the curve plotted from the real mortality data for the study population is within the limits of the confidence interval that had been adopted $(\alpha=0.05 \%)$.

\subsection{Case Studies}

Figure 5 presents results of the proposed model for the case study 1 with the following characteristics: female, mild high blood pressure (that does not interfere with her life), free from diabetes, not obese and does not practice physical activity. Data on smoking and cholesterol not given. Observing this figure, it will be noted that the mortality rate defines 3 distinct age groups: (1) from 60 to 69 the mortality rate is low, at less than 7\%; (2) from 70 to 79 the rate is between 9 and 21\%; and (3) over 80 years of age, the mortality rate is between 80 and $85 \%$.

Figure 6 presents the results of the proposed model for the case study 2 with the following characteristics: female, free from high blood pressure, free from diabetes, not obese and practices physical activities 3 or more times per week. Data on smoking and cholesterol not given. Observing this figure, it will be noted that the mortality rate in the first age group (from 60 to 69 years) is practically negligible, at below $4 \%$. In the age group from 70 to 79 , the mortality rate is between 2 and $13 \%$ and, finally, in the age group over 80 years mortality is from 84 to $99 \%$.

Figure 7 presents results of the proposed model for the case study 3 with the following characteristics: male, free from high blood pressure, free from diabetes, not obese and practices physical activities 3 or more times per week. Data on smoking and cholesterol not given. Observing this figure, it will be noted that the mortality rate in the age group from 60 to 69 years is below 7\%. In the age group from 70 to 79 , the mortality rate is between 19 and $33 \%$ and, finally, in the age group over 80 years mortality is from 63 to $79 \%$.

Case studies 1 and 2 differ in terms of the characteristics high blood pressure and physical activity level. A comparison of the results (Figures 5 and 6) reveals that the mortality rate is lower in case study 2 in the first two age groups with relation to case study 1 . This observation leads to the conclusion that maintaining good blood pressure and participating in physical activities have an impact on a person's lifespan, delaying mortality in the population. Case studies 2 and 3 differ in terms of the sex of the population. A comparison of the results (Figures 6 and 7) reveals that the mortality rate is higher in case study 3 than case study 2 in 


\section{Ferranti and Freitas Filho}

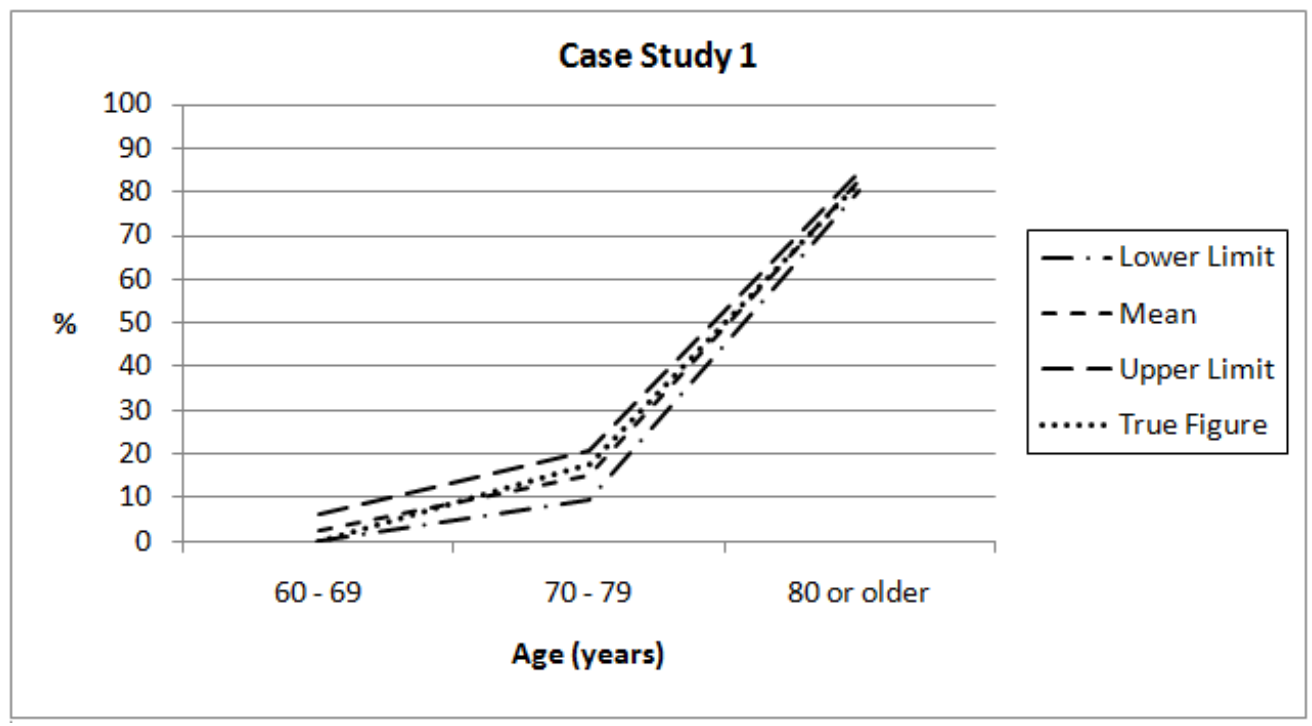

Figure 5: Results of the proposed model for a case study with the following parameters: $G R=0.14$, $A D F=4, R R=0.05, A I R=100$ and $R A=0.021$.

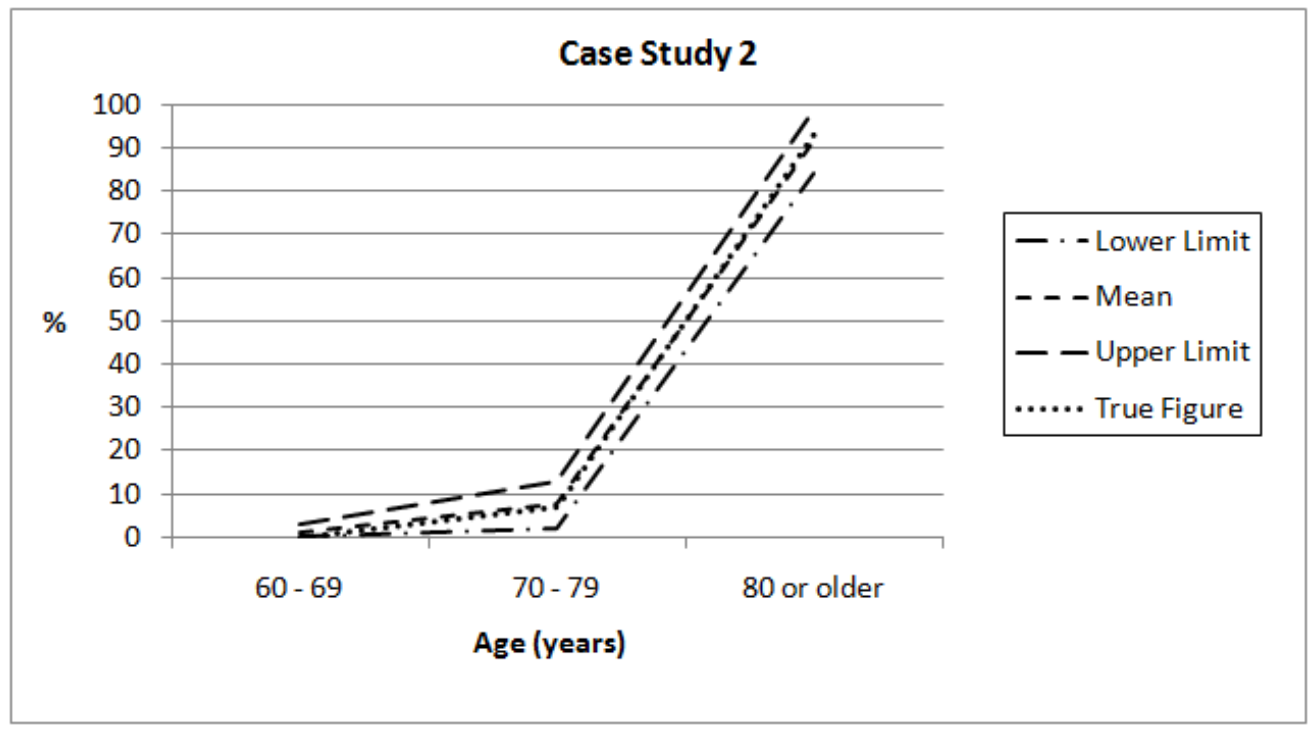

Figure 6: Results of the proposed model for a case study with the following parameters: $G R=0.14$, $A D F=4, R R=0.42, A I R=100$ and $R A=0.017$. 


\section{Ferranti and Freitas Filho}

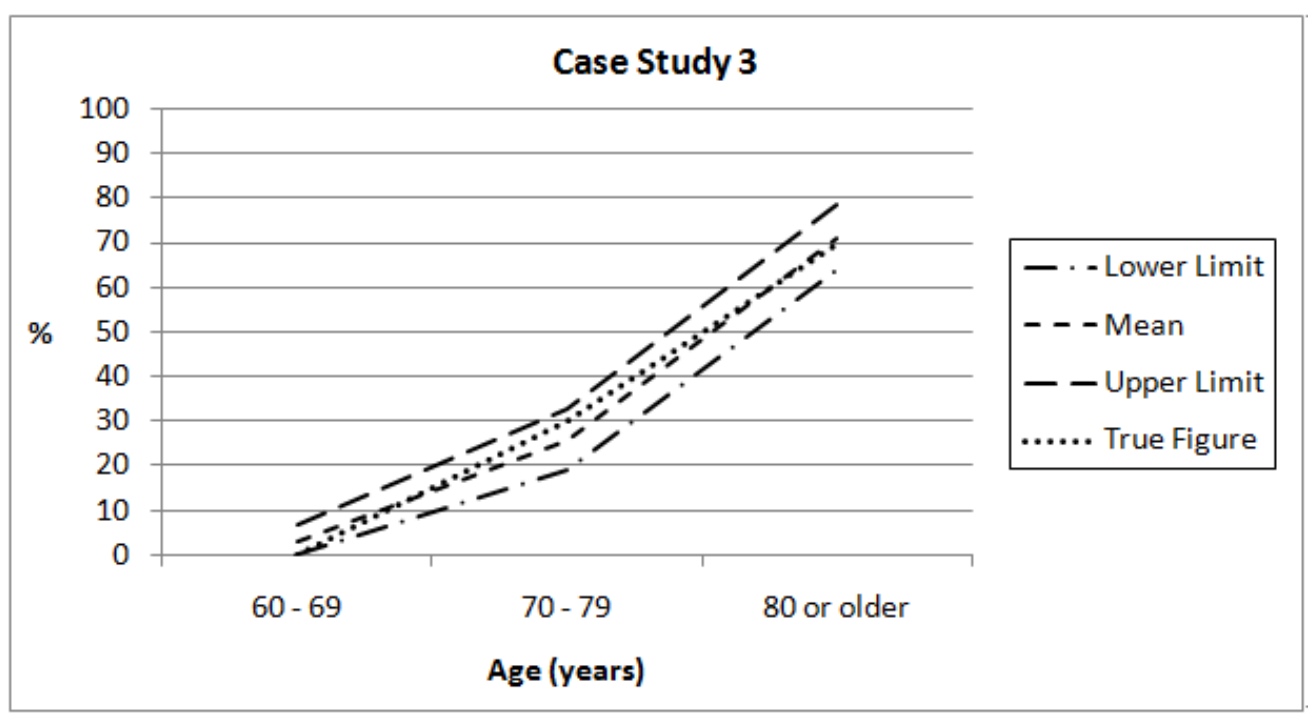

Figure 7: Results of the proposed model for a case study with the following parameters: $G R=0.14$, $A D F=4, R R=0.03, A I R=100$ and $R A=0.022$.

the second age group. This observation leads to the conclusion that sex is related to a person's lifespan, anticipating mortality in the male population.

\section{CONCLUSIONS}

This article has both described and proposed an extension to a dynamic-fuzzy simulation model which represents a person's physiological functions throughout life and expresses the occurrence of risk events which may occur in the form of accidents or as a result of advancing age.

The model on which the expanded model is based (Schwaab and de Freitas Filho 2009) was originally used to reproduce mortality curves for the general population of the Brazilian state of Santa Catarina and so it did not take into account specific risk factors that can either exacerbate or attenuate the incidence of diseases that are causes of death within the population, nor the fact that appropriate changes to factors related to lifestyle habits can both avoid and reverse conditions that aggravate diseases, such as hypertension, cholesterol and diabetes.

The proposal described here was therefore to refine the earlier model by including specific risk indicators for cardiovascular diseases in order to calculate the impact of these indicators on the probability of death of people who share certain characteristics. The results generated by the model were validated against mortality curves plotted with data from a prospective epidemiological study provided by the EPIDOSO project (Ramos 1998) and mortality data from São Paulo's SEADE Foundation (SEADE 2011). The model proved capable of reproducing real-life mortality data curves for populations considered to be homogeneous in terms of characteristics such as hypertension, diabetes, obesity and physical activity level.

Constructing a simulation model that deals with the aging process and with the risk factors associated with causes of death is one means of achieving a better understanding of the factors involved in the process, thereby facilitating the planning of strategies to attenuate the incidence of these diseases through the adoption of appropriate lifestyle habits. In contrast, validation of such strategies using real populations can only be achieved through long-term follow-up studies.

\section{ACKNOWLEDGMENTS}

We would like to thank Dr. Luiz R. Ramos, Dr. André J. Xavier and Dr. Eleonora d'Orsi for providing the data use to validate the model and for their support for this research. Text translated by Robert Coulthard. 


\section{Ferranti and Freitas Filho}

\section{REFERENCES}

Deaton, M. L., and J. J. Winebrake. 2000. Dynamic Modeling of Environmental Systems. New York: Springer-Verlag.

Ford, A. 1999. Modeling the Environment: An Introduction to System Dynamics Modeling of Environmental Systems. Washington: Island Press.

Forrester, J. W. 1961. Industrial Dynamics. Waltham: Productivity Press.

Forrester, J. W. 1969. Urban Dynamics. Waltham: Pegasus Communications, Inc.

Forrester, J. W. 1998. "Designing the future". Presented at Universidad de Sevilla.

Hall, J. H., and J. D. Zwemer. 1979. Prospective Medicine. Indianapolis, Indiana: Methodist Hospital of Indiana.

Hammersley, J. M., and D. C. Handscomb. 1964. Monte Carlo Methods. London: Methuen.

Hargrove, J. L. 1998. Dynamic Modeling in the Health Sciences. New York: Springer-Verlag.

Hayflick, L. 1997. Como e Por Que Envelhecemos. Rio de Janeiro: Campus.

isee Systems 2011. Stella Modeling \& Simulation Software. isee Systems.

Klir, G. J., and B. Yuan. 1995. Fuzzy Sets and Fuzzy Logic: Theory and Applications. New Jersey: Prentice Hall.

Radzicki, M. J. and Taylor, R. A. 1997. "Introduction to system dynamics: A systems approach to understanding complex policy issue". http://www.systemdynamics.org/DLIntroSysDyn/inside.htm.

Ramos, L. R. 1998. "Two-year follow-up study of elderly residents in S. Paulo, Brazil: methodology and preliminary results". Revista Saúde Púplica 5:397-497.

Schwaab, A. A., and P. J. de Freitas Filho. 2009, December. "Dynamic-Fuzzy Simulation Model for Reproduction of Mortality Curves". In Proceedings of the 2009 Winter Simulation Conference, edited by M. D. Rossetti, R. R. Hill, B. Johansson, A. Dunkin, and R. G. Ingalls, 2078-2089. Piscataway, New Jersey: Institute of Electrical and Electronics Engineers, Inc.: Institute of Electrical and Electronics Engineers, Inc.

SEADE 2011. "Fundação Sistema Estadual de Análise de Dados". Accessed Mar. 2, 2011. http://http: //www.seade.gov.br.

Zadeh, L. A. 1965. "Fuzzy Sets". Information and Control 8:338-353.

\section{AUTHOR BIOGRAPHIES}

JOCIMARA SEGANTINI FERRANTI is a masters student on the Postgraduate program in Computer Science at the Federal University of Santa Catarina (UFSC). She has a bachelor's degree in Information Systems Development Technology from the Federal Technological University of Paraná (UTFPR) in 2006 and her research interests are modeling and simulation and artificial intelligence. Jocimara's email address is joccyferranti@gmail.com.

PAULO JOSÉ DE FREITAS FILHO graduated from the Federal University of Rio Grande do Sul in 1978 with a degree in Metallurgical Engineering, completed a Masters in Production Engineering at the Federal University of Santa Catarina (UFSC) in 1985 and his doctorate in Systems Engineering was granted by UFSC and the University of South Florida in 1994. He is currently a Professor at UFSC. His experience is centered on Computer Science with an emphasis on Systems Performance and his teaching and research is primarily in the following areas: modeling and simulation, performance assessment and systems capacity planning. Over the past four years he devoted much of his time to research on modeling and simulation applied to health care. Paulo's e-mail is freitas@inf.ufsc.br. 